\title{
Comparative analysis of methods and results of numerical calculations of plywood panel
}

\author{
Irene Ladnykh *1, Alexander Ibragimov ${ }^{1}$ \\ ${ }^{1}$ Moscow State University of Civil Engineering, Yaroslavskoye shosse 26, Moscow, \\ 129337 Russian Federation
}

\begin{abstract}
The article discusses the new type of plywood panel for lowrise building. Calculating methods for the plywood panels are considered according with the codes of rules and the method for calculating sandwich panels with a honeycomb, proposed by Soviet scientists for aviation structures.
\end{abstract}

\section{Introduction.}

In the post-Soviet space, the plywood panels (figure 1) are widely used as wall and overlapcovering panels. They first appeared in the 40s of the XX century. The development of necessary computational formulas and reliable data on anisotropy was carried out by such scientists as E.K. Ashkenazi [1], E.V. Ganow, I.M. Linkov [2], V.M. Khrulev and others. The design of these panels is a wooden supporting frame made of timber, connected with plywood linings with glue. The main advantage of such panels is a high degree of factory readiness, but a significant disadvantage is the low degree of form stability of the main carrying ribs.

\footnotetext{
${ }^{1}$ Corresponding author: irenlad@yandex.ru
} 

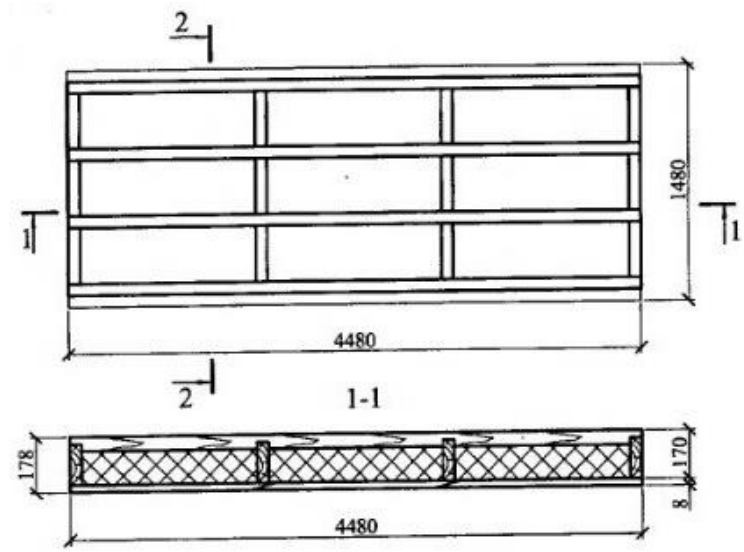

$2-2$

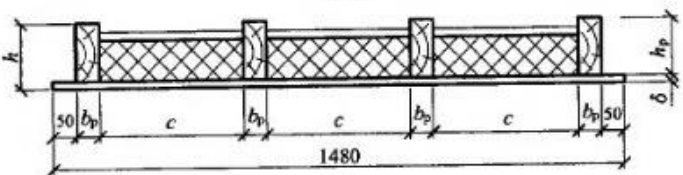

Fig. 1 - The forms of plywood panel

In the late sixties and early seventies of the twentieth century another direction appeared in the wooden panel building based on layered structures. A huge contribution to its development was made by such scientists as V.A. Ivanov and B.E. Shungsky [3]. Laminated structures are a composition of two skins and a honeycomb core, connected with each other by glue, welding or soldering. The shape of the cells can be different, but the most common are hexagonal. Examples of honeycomb forms are shown in Figure 2.
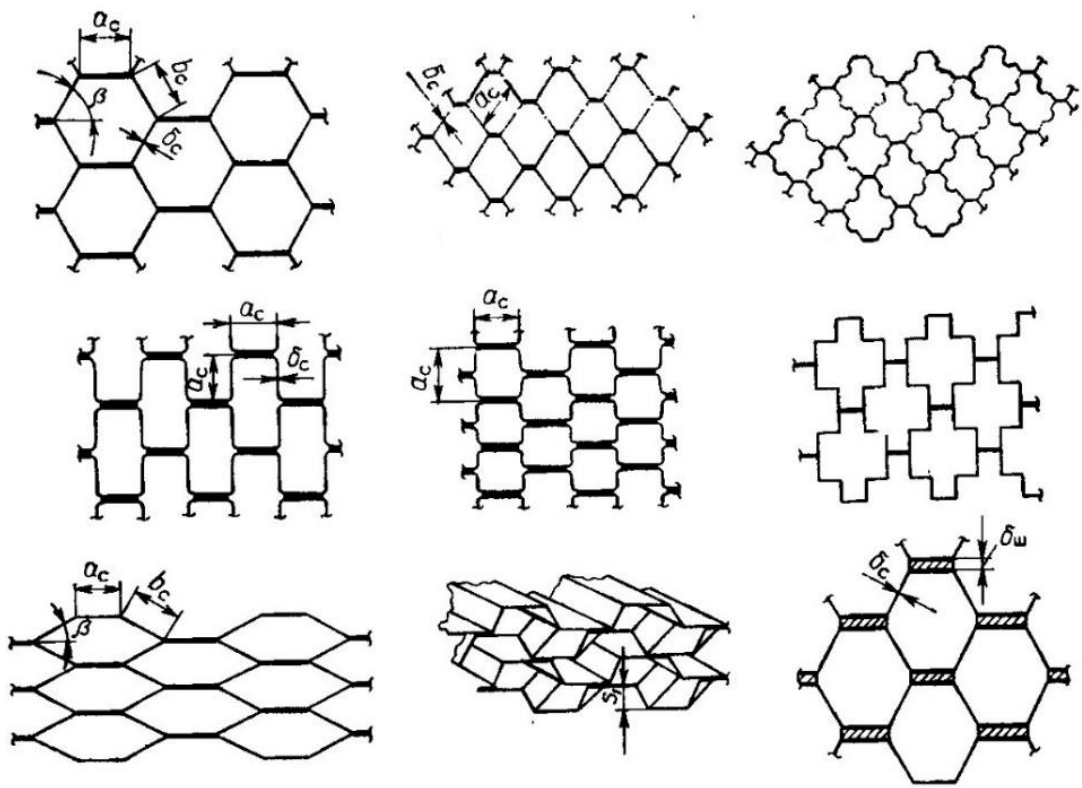

Fig. 2 - The forms of honeycomb

The works of B.E. Shungsky and V.A. Ivanova considers a wood-paper honeycomb core with a hexagonal cell (Figure 3). 

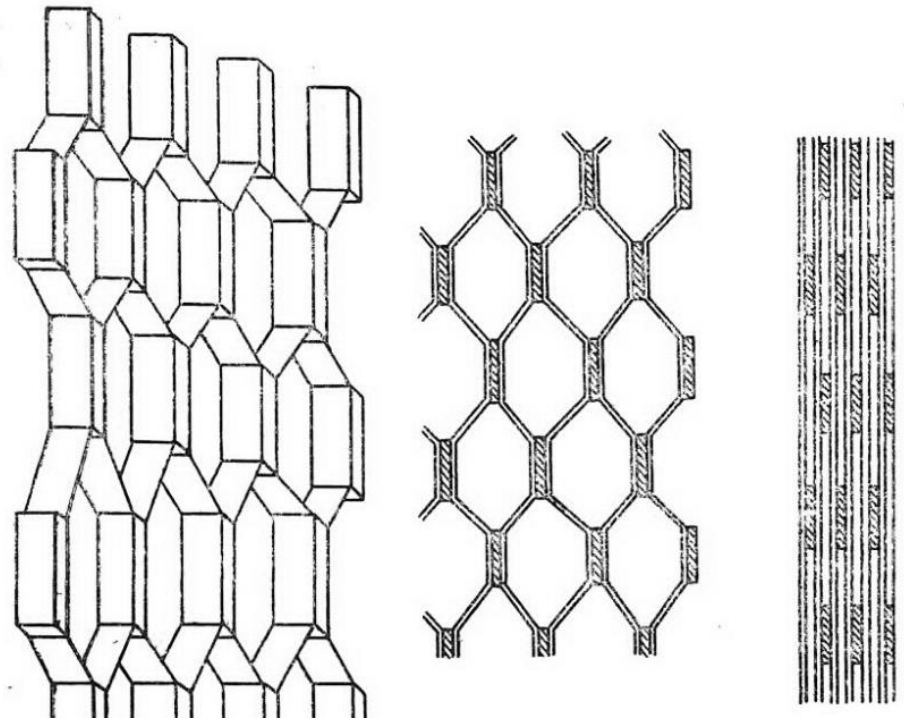

Fig. 3 - Example of a wood-and-paper honeycomb

Based on the research data in 1968 at VDNH (Moscow) was presented the first garden house made of panels with a honeycomb core (Figure 4).

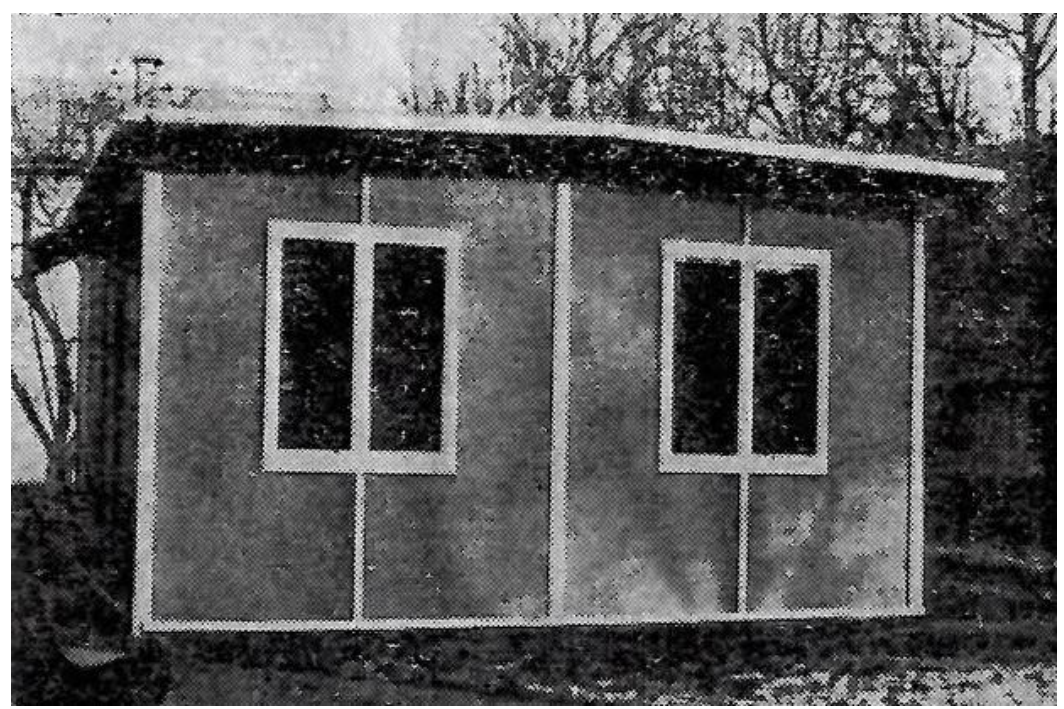

Fig. 4 - Garden house made of panels with honeycomb - an exhibit of the USSR Exhibition of National Economic Achievements (1968)

The main disadvantages of the sandwich panels with honeycomb in the form of a hexagon is low form stability in a direction perpendicular to the position of the cells.

The authors of this article are invited to consider a new type of plywood panels with honeycomb for use in low-rise wooden housing construction, presented in Figure 6 [4]. This type of panel has several advantages in comparison with the panels presented above: a high degree of form stability. The cell is based on a triangle. However, at present, an 
obstacle to the widespread use of such panels is the lack of a methodology for calculating panels with a triangular cell.

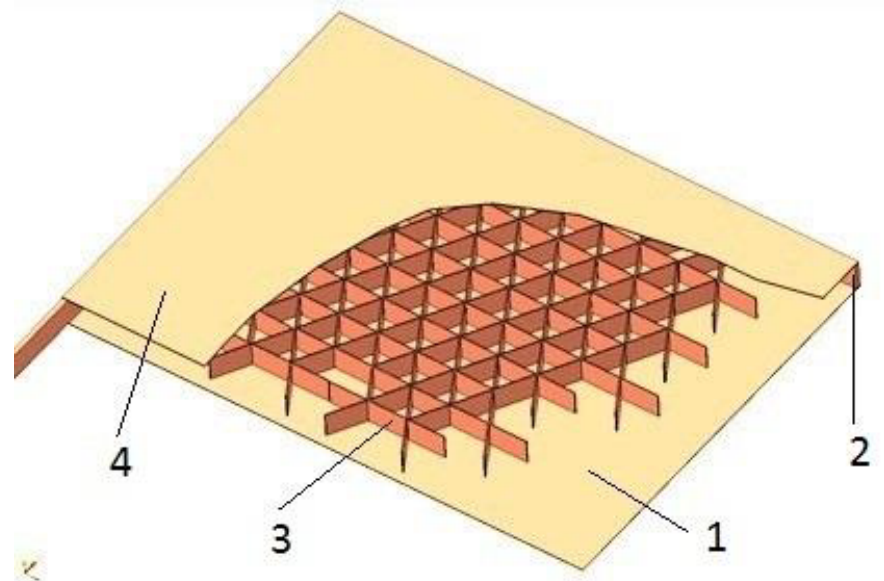

Fig. 6 - The form of new type panel (1 - exterior covering; 2 - bundling beam; 3 - honeycomb; 4 interior covering)

The height and width of the overlapping panels are determined by the architectural and construction requirements and the format of the sheathing sheets. The thickness of the edges is regulated by the thickness of the plywood, the thickness of the skin at the junction is equal to the thickness of the plywood except for one or two layers of veneer.

Due to the fact that the panels are intended to be used in the construction industry, the requirements, as well as structural loads, will therefore be accepted in accordance with the regulatory documents in force in the territory of the Russian Federation - a set of rules. Layered structures are calculated from two groups of limiting states: by their carrying capacity (strength or stability) and by deformation. When calculating the second limiting state, the ultimate deflections of the structures are taken in accordance with the values, given in Appendix E [5] and are equal to 1/125.

Table 1 presents the numerical values of the panel's geometric characteristics, which will be used for further calculations with a view to performing a comparative analysis of the methods for calculating three-layered plywood panels with a honeycomb core.

Table 1 - General parameters of the panel-overlapping.

\begin{tabular}{|c|c|l|c|}
\hline$\#$ & Symbol & Name & $\begin{array}{l}\text { The numerical value for the } \\
\text { example, unit. }\end{array}$ \\
\hline 1 & $l$ & Panel length & $6100 \mathrm{~mm}$ \\
\hline 2 & $b$ & Panel width & $1525 \mathrm{~mm}$ \\
\hline 3 & $t$ & Panel thickness & $140 \mathrm{~mm}$ \\
\hline 4 & $\delta \mathrm{f}^{\mathrm{o}}$ & Thickness of plywood skin & $7 \mathrm{~mm}$ \\
\hline 5 & $\delta_{\mathrm{f}}^{\mathrm{r}}$ & Thickness of plywood ribs & $8 \mathrm{~mm}$ \\
\hline
\end{tabular}

The value of the payload will be equal to $1.5 \mathrm{kPa}$, the load reliability factor is assumed to be equal to 1.3 according to [5]. When calculating the second limit state (in terms of deformations), the ultimate deflections of the structures are taken in accordance with the values given in Appendix E [5] and are 1/125.

Methods. In this article, we will consider two different methods for calculating sandwich plywood panels with a honeycomb.

1. Calculation using the methodology of code of rules 64.13330 "Wooden structures" 
The normative documents $[6,7,8,9]$ give recommendations for the design and calculation of the glued-up panels. In [6], Section 6 describes the approach to the calculation of glued elements from plywood with wood, where the main method is the method of reduced crosssection. No matter how the panel is cut in the width direction, the same number of edges will fall into the section. To simplify further mathematical calculations and calculations, we introduce an additional value of $n$, which will be called the number of plywood ribs in the air gap over the width of the common $b^{\prime}$.

$$
n=2 \cdot \frac{b^{\prime}-\frac{a}{2} \cdot \tan \alpha}{\delta_{\mathrm{f} \phi}^{\mathrm{r}}+\frac{a}{2} \cdot \tan \alpha}+1
$$

According to the technique, we calculate the geometric characteristics: the moment of inertia, the moment of resistance, the static moment with respect to the neutral axis. The moment of inertia of a given section will be:

$$
I_{\mathrm{pr}}=I_{f}+n \cdot I_{\mathrm{f}}^{\mathrm{r}}+2 \cdot I_{\mathrm{br}} \cdot \frac{E_{\mathrm{dr}}}{E_{\mathrm{f}}}
$$

where $I_{\mathrm{f}}-$ moment of inertia of plywood panels skin

$I_{f}^{\mathrm{r}}-$ moment of intertia of plywood panels ribs

$I_{\mathrm{br}}-$ moment of intertia of beam

$E_{\mathrm{dr}}-$ elastic modulus of timber

$E_{f}$ - elastic modulus of plywood.

Table 2 shows the values of the geometrical characteristics of the cross section.

Table 2 - Geometrical characteristics of a section

\begin{tabular}{|c|c|l|c|}
\hline$\#$ & Symbol & Name & $\begin{array}{c}\text { The numerical value for the } \\
\text { example, unit }\end{array}$ \\
\hline 1 & $I_{p r}$ & Moment of inertia & $14010 \mathrm{sm}^{4}$ \\
\hline 2 & $W_{p r}$ & Resistance moment & $2002 \mathrm{sm}^{3}$ \\
\hline 3 & $S_{p r}$ & Static moment & $811 \mathrm{sm}^{3}$ \\
\hline
\end{tabular}

We perform the basic verification calculations:

1. For the first group of limiting states:

1.1. Verification of the strength of the expanded plywood paneling of panels and panels, according to paragraph 6.25 [6].

1.2. Check for the stability of the compressed sheathing of slabs and panels in accordance with paragraph 6.28 [6].

1.3. Check for the chipping of the edges of the frame of panels and panels or sheathing along the seam at the junction with the ribs.

2. For the second group of limiting states:

2.1. Check the panel for the ultimate deflection.

The main results of the calculation are presented in Table 3.

\section{Calculation by the method of calculating the honeycomb aggregate}

The second method, which can be applied to the calculation, is the calculation of layered structures, based on the theory of A.Ya. Aleksandrov set forth in the works $[10,11,12]$.

The main condition for calculating three-layer structures with a honeycomb core is the knowledge of the mechanical characteristics of the honeycomb core itself. Therefore, the reduced mechanical characteristics of the aggregate are first determined, which is a kind of conditional solid material endowed with all the properties of a real honeycomb core, and occupying the same volume as the honeycomb core itself. 
In our case, let us single out the typical element of the honeycomb block, presented in Figure 7, for which we will determine the reduced mechanical characteristics.
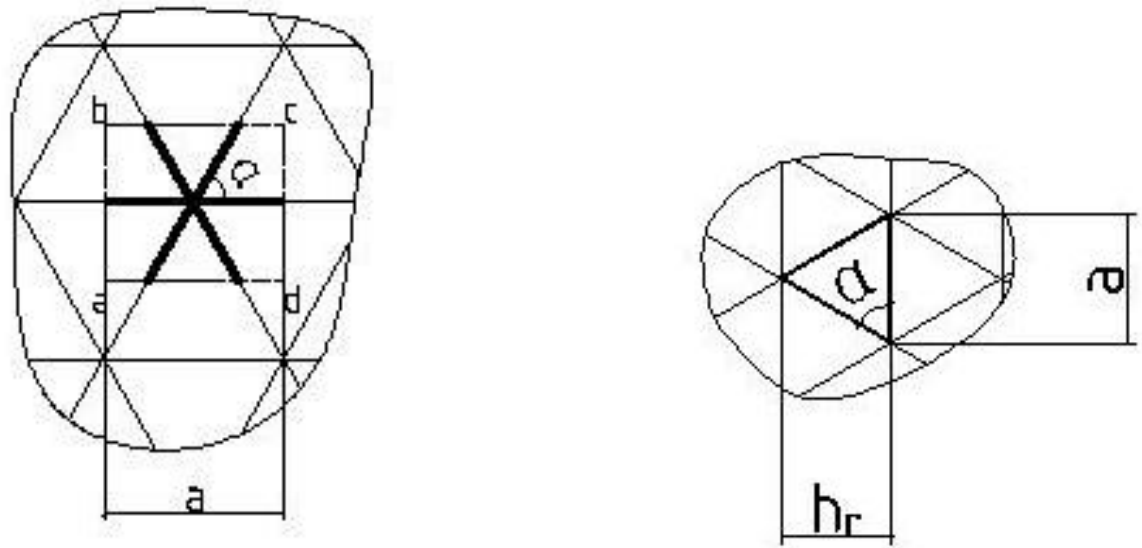

Fig. 7 - A typical honeycomb element of panel

The main mechanical characteristics are: the module of normal elasticity $\mathrm{E}_{\mathrm{Z}}$; Shift modules $\mathrm{G}_{\mathrm{XY}}, \mathrm{G}_{\mathrm{YZ}} ;$ Allowable voltages $\left[\sigma_{\mathrm{Z}}\right],\left[\tau_{\mathrm{XZ}}\right],\left[\tau_{\mathrm{YZ}}\right]$. It should be noted that the most difficult is to determine the shear moduli. Until now, there is not a single technique that could accurately reflect the actual value of the shear modulus, so an approximate value is taken in the calculations or the value is taken from the experimental data for the honeycomb.

Further:

1. Determine the critical stress loss of general and local stability for an ideally manufactured plate.

The deflection of the plate is determined as follows:

$$
f_{m n}=\frac{\omega=\sum_{n=1}^{n} \sum_{m=1}^{m} f_{m n} \sin \frac{m \pi x}{l} \sin \frac{n \pi y}{b}}{\pi^{6} m n\left\{D\left(m^{4}+2 \frac{m^{2} n^{2}}{\psi_{L}^{2}}+\frac{n^{4}}{\psi_{L}^{4}}\right)-N_{x}\left[\frac{l^{2}}{\pi^{2}}+\frac{D}{h G_{x Z}\left(m^{2}+\frac{n^{2}}{\psi_{L}^{2}}\right)}\right] m^{2}\right\}}
$$

where $\mathrm{m}=1,3,5, \ldots,(2 \mathrm{k}-1) ; \mathrm{k}=1,2,3, \ldots ; \mathrm{n}=1,3,5, \ldots,(2 \mathrm{r}-1) ; \mathrm{r}=1,2,3, \ldots ; \psi_{L}=\frac{b}{l}$

The maximum values of the bending moments (in the center of the plate):

$$
\begin{aligned}
& M_{x \max }=\sum_{m=1}^{m} \sum_{n=1}^{m} f_{m n} \frac{\pi^{2} D}{l^{2}}\left(m^{2}+\mu^{2} \frac{n^{2}}{\psi_{L}}\right) \sin \frac{m \pi}{2} \sin \frac{n \pi}{2} \\
& M_{y \max }=\sum_{m=1}^{m} \sum_{n=1}^{m} f_{m n} \frac{\pi^{2} D}{l^{2}}\left(\mu m^{2}+\frac{n^{2}}{{\psi_{L}}^{2}}\right) \sin \frac{m \pi}{2} \sin \frac{n \pi}{2}
\end{aligned}
$$

Maximum shearing forces (on the support contour in the middle of the support edges):

$$
\begin{aligned}
& Q_{x \max }=\sum_{m=1}^{m} \sum_{n=1}^{m} f_{m n} \frac{\pi^{3} D}{l^{3}}\left(m^{2}+\frac{n^{2}}{{\psi_{L}}^{2}}\right) \sin \frac{n \pi}{2} \\
& Q_{y \max }=\sum_{m=1}^{m} \sum_{n=1}^{m} f_{m n} \frac{\pi^{3} D}{l^{3}}\left(m^{2}+\frac{n^{2}}{{\psi_{L}}^{2}}\right) \sin \frac{m \pi}{2}
\end{aligned}
$$

Maximum normal stresses in the bearing layers of the plate:

$$
\begin{gathered}
\sigma_{1 x}=\frac{M_{x \max }}{H \delta_{1}}+\frac{N_{x}}{\delta_{1}\left(1+\psi_{\delta}\right)} \\
\sigma_{2 x}=\frac{M_{x \max }}{H \delta_{1} \psi_{\delta}}-\frac{N_{x}}{\delta_{1}\left(1+\psi_{\delta}\right)} \\
\sigma_{1 y}=\frac{M_{y \max }}{H \delta_{1}} \\
\sigma_{2 y}=\frac{M_{y \max }}{H \delta_{1} \psi_{\delta}}
\end{gathered}
$$


where $\psi_{\delta}=\frac{\delta_{2}}{\delta_{1}} ; \delta 1$ - thickness of a carrier layer compressed under bending; $\delta 2$ - Thickness of the bending layer.

The maximum tangential stresses in the honeycomb:

Checking the strength of the honeycomb:

$$
\begin{aligned}
& \tau_{x \text { zmax }}=\frac{Q_{x \max }}{h} \\
& \tau_{y \text { max }}=\frac{Q_{y \max }}{h}
\end{aligned}
$$

$$
\frac{\sigma_{z}}{\left[\sigma_{z}\right]}+\left(\frac{\tau_{x z}}{\left[\tau_{x z}\right]}\right)^{2} \leq 1
$$

2. Determine the forms of general and local loss of stability.

3. Given initial imperfections, a further calculation is carried out to determine the bearing capacity for wide plates.

Results and Discussion. The main results of the calculation are presented in Table 3.

Table 3 - Calculation results

\begin{tabular}{|c|c|c|c|}
\hline$\#$ & Symbol & The method \#1 & The method \#2 \\
\hline 1 & $\sigma$ & $6.796 \mathrm{MPa}$ & $5.874 \mathrm{MPa}$ \\
\hline 2 & $f$ & $32.0 \mathrm{~mm}$ & $24.8 \mathrm{~mm}$ \\
\hline
\end{tabular}

Conclusions. The analysis of methods and approaches to calculation, stated above, allows us to draw the following conclusions:

1. The procedure for calculating the glued panel according to [6] is simpler, and the calculation can be performed manually.

2. Calculation using the procedure $[10,11,12]$ is more time-consuming, requires a large amount of experimental data or the introduction of approximate values, which must be further confirmed by experimental data.

3. Comparing the results of the calculations presented in Table 3, we note that the value of normal stresses and deflection is greater when calculated by the method proposed in the JV. This is explained by the fact that the second technique takes into account the greater number of parameters, both geometric and mechanical.

For the final choice of calculation methods, the authors suggest, compare the results obtained with the results of computer simulation and full-scale experiment.

\section{References}

1. E. K. Ashkenazi, Anisotropy of wood and wood materials. - Moscow: Forest Industry, (1978)

1. I.M. Linkov, Development and research of structures of glued plywood reinforced panels. Tr. CNIISK them. V.A. Kucherenko. Moscow (1972)

2. B.E. Shungsky, Building structures with honeycomb aggregates. - M.: Stroiizdat, (1977)

3. Patent of the Russian Federation No. 160223. Panel / Tikhomirov LA, Zaitseva KV, Titunin AA, Ibragimov AM, Gnedina L.Yu ; Declared. 07/20/2015. Opubl. 03/10/2016.

4. SP 20.13330.2011 "Loads and impacts. Updated version of SNiP 2.01.07-85 * ». Moscow: FGUP TsPP, (2011)

5. SP 64.13330.2011 "Wooden structures. Updated version of SNiP II-25-80. Moscow: FGUP TsPP, (2011)

6. Manual on the design of wooden structures (to SNiP II-25-80) / TsNIISK im.Kucherenko // Moscow: Stroiizdat, (1986) 
7. Recommendations on the design of panel structures using wood and wood materials for industrial buildings) / TsNIISK im.Kucherenko // M .: Stroiizdat, (1982)

8. Guide to the design of glued wooden structures. / TsNIISK im.Kucherenko // Moscow: Stroyizdat, (1977)

9. A. Ya. Alexandrov, E.P. Trofimova, Determination of the reduced elastic parameters of honeycomb aggregates: coll. Questions of calculating the elements of aviation structures. - Moscow: Oborongiz, (1959)

10. A.I. Endogur, M.V. Weiberg, Jerusalem KM Cellular constructions. Selection of design parameters. - M.:, Mechanical Engineering (1986)

11. M. Ya. Gofin, A.A. Ivanov, Mechanics of honeycomb structures: reference book in 2 volumes. - M .: TF "Mir" (2012) 